\title{
DETECTION OF AN ELECTRON SPIN IN A MRFM CANTILEVER EXPERIMENT
}

\author{
Michael Ting and Alfred O. Hero \\ Dept. of Electrical Engineering \& Computer Science \\ University of Michigan, Ann Arbor, MI 48109
}

\begin{abstract}
In the Magnetic Resonance Force Microscopy (MRFM) experiment that we consider, a cantilever is used to detect the presence of an electron spin. If an electron spin is present, the equation governing the motion of the cantilever is nonlinear. In the absence of a spin, the equation is linear. We propose a detector model that uses two Kalman Filter (KF) state estimators. The estimator for the spin hypothesis is an adaptation of the standard $\mathrm{KF}$, and can be viewed as a 0th order mixed-time Extended Kalman Filter (EKF). Simulation results are presented for several system parameter values.
\end{abstract}

\section{INTRODUCTION}

The principle of magnetic resonance has been successfully applied in the imaging of human tissue. A technique that attempts to extend this to the detection of a single-spin electron has been proposed in $[1,2]$, and is known as Magnetic Resonance Force Microscopy (MRFM). The proposed experiment makes use of the interrupted Oscillating Cantileverdriven Adiabatic Reversal (OSCAR) idea. An oscillating cantilever with a magnetic tip is brought close to an electron spin. The movement of the cantilever causes the spin to cycle in and out of magnetic resonance. In the normal mode of operation of interrupted OSCAR, the electron spin is "spinlocked", and the $z$ component of the spin follows the motion of the cantilever. The spin functions as a small magnet, and its presence will modify the cantilever's frequency of oscillation $\omega_{0}$. Detection of the electron is equivalent to detecting the minute frequency changes in $\omega_{0}$ of the cantilever position signal $z(t)$. A straight-forward detection method is to pass $z(t)$ through a Frequency Modulation (FM) demodulator, and looking for the expected behaviour. Another baseband method of detecting this frequency shift is given in [3]; however, this method makes an assumption on the form of the baseband output. In this paper, we propose a more direct detection scheme that uses samples of the cantilever's position, and is based on the well-known Kalman Filter (KF) algorithm [4], [5]. It is hoped that better de-

Research partially funded by the DARPA Mosaic Program under ARO contract DAAD19-02-C-0055 tection performance can be achieved by using $z(t)$ directly without any additional assumptions.

\section{PROBLEM DESCRIPTION}

Let $H_{0}$ be the hypothesis that no spin is present, and $H_{1}$ that a spin is present. Let $\Sigma_{0}$ and $\Sigma_{1}$ be the systems under $H_{0}$ and $H_{1}$ respectively. $\Sigma_{0}$ is the well-known linear, 2 nd order system, of a moving cantilever. The equations describing $\Sigma_{0}$ are given below, and a description of the pertinent variables follows. Note that an overhead dot is understood to be differentiation with respect to time. That is, $\dot{\square}=\frac{d}{d t} \square$.

$$
\begin{aligned}
m \ddot{z}+\Gamma \dot{z}+k z & =F_{n}(t) \\
x[i] & =z\left(t_{i}\right)+\zeta[i]
\end{aligned}
$$

$z(t)$ : Cantilever position (equilibrium position is $z=0$ )

$F_{n}(t)$ : Thermal noise, assumed to be White Gaussian Noise (WGN)

$t_{i}: i$ th sampling time instant, where $t_{i}=i T_{s}\left(T_{s}\right.$ is the sampling period)

$x[i]: i$ th observed sample of $z(t)$

$\zeta[i]$ : Observation noise, assumed to be a sequence of i.i.d. Gaussian r.v.'s with mean 0 and variance $\sigma^{2}$

The equations of the classical dynamics of a MRFM cantilever interacting with a single electron spin moment are described in [6]. If one considers only the fundamental mode and takes $F_{\text {control }}(t)=0$, the evolution of $z(t)$ in $\Sigma_{1}$ is described by the nonlinear system:

$$
\begin{aligned}
\dot{\mu}_{x} & =\gamma \mu_{y}\left(G z+\delta B_{0}\right) \\
\dot{\mu}_{y} & =\gamma \mu_{z} B_{1}(t)-\gamma \mu_{x}\left(G z+\delta B_{0}\right) \\
\dot{\mu}_{z} & =-\gamma \mu_{y} B_{1}(t) \\
m \ddot{z}+\Gamma \dot{z}+k z & =G \mu_{z}+F_{n}(t)
\end{aligned}
$$

$\vec{\mu}$ : Electron spin moment, which is composed of $\vec{\mu}=$ $\left[\mu_{x} \mu_{y} \mu_{z}\right]$ 
$B_{1}(t)$ : Radio frequency (rf) signal that brings the spin moment into resonance. This is a control input to the system.

Similar to $\Sigma_{0}$, one only observes samples of $z(t)$ at multiples of $T_{s}$. The detection problem is as follows: given $N$ noisy observations $x[i], i=1, \ldots, N$, classify the system which generated the $x[i]$ 's as either $H_{0}:\{x[i]\}_{i=0}^{N}$ generated by $\Sigma_{0}$, or $H_{1}:\{x[i]\}_{i=0}^{N}$ generated by $\Sigma_{1}$. For reasons relating to the practical implementation of the detector, we shall assume a low sampling frequency $f_{s}=1 / T_{s}$. As well, we shall assume that the number of observations $N$ is small.

\section{ANALYSIS OF $\Sigma_{1}$}

$\Sigma_{1}$ is a stiff nonlinear system. The electron spin moment $\vec{\mu}$ has a constant 2 -norm that is given by $|\vec{\mu}|=9.28 \times 10^{-24}$ $\mathrm{J} / \mathrm{T}$. The invariance of $|\vec{\mu}|$ can be verified by considering $V\left(\mu_{x}, \mu_{y}, \mu_{z}\right)=\mu_{x}^{2}+\mu_{y}^{2}+\mu_{z}^{2} \Rightarrow \dot{V}=0$ by using the expressions for $\dot{\mu}_{x}, \dot{\mu}_{y}$, and $\dot{\mu}_{z}$ in (2). Setting $B_{1}(t) \equiv 0$ in the equations for $\Sigma_{1}$ enables us to solve for $\mu_{x}(t)$ and $\mu_{y}(t)$. We obtain the solution $C \cdot \cos \left[-\gamma G \int_{0}^{t} z(\tau) d \tau+\theta\right], C$ and $\theta$ being some constants which are different for $\mu_{x}$ and $\mu_{y}$. Since $\left|\gamma G z_{a m p l}\right|$ is a large quantity (let $z_{a m p l}$ be the amplitude of $z(t)$ ), the $\mathrm{x}$ and y components of $\vec{\mu}$ are oscillating very rapidly. With a non-zero $B_{1}(t)$, the same holds true for the simulated system. As a result, a small integration time step is required, on the order of $10^{-10}$ to $10^{-12}$ seconds.

Nonlinear systems analysis methods can be applied to analyze the reachability and observability properties of $\Sigma_{1}$ $[7,8] . \Sigma_{1}$ can be written in state-space form by defining the state vector $v(t)=\left[\begin{array}{lllll}\mu_{x} & \mu_{y} & \mu_{z} & z & \dot{z}\end{array}\right]^{T}$ and $w(t)=$ $\left[0 \ldots 0 F_{n}(t) / m\right]^{T}$. Then, from (2), a suitable function $f: \mathbf{R}^{5} \times[0, \infty) \rightarrow \mathbf{R}^{5}$ can be defined so that $v(t)$ evolves according to $\dot{v}=f(v, t)+w(t)$. The time dependency in $f(v, t)$ arises from $B_{1}(t)$, the if signal. If we treat $u=$ $\left[B_{1}(t) F_{n}(t)\right]$ as the control inputs, $\Sigma_{1}$ assumes the form of a linear-analytic system. That is, we can write $\dot{v}=$ $p(v)+g(v) u$ for suitable functions $p(\cdot)$ and $g(\cdot)$. In this formulation, $\Sigma_{1}$ is locally observable everywhere except for the set of points $\Omega_{1}$, where $\Omega_{1}=\left\{v: G v_{4}+\delta B_{0}=0\right\} . \Sigma_{1}$ is not locally reachable. Instead, it is locally reachable on a sub-manifold of dimension 4 at almost all points except for those which are in either $\Omega_{1}$ or $\Omega_{2}=\left\{v: v_{3}=0\right\}$. Note, however, that the solution manifold of $\Sigma_{1}$ is of dimension 4 , as $\left[\begin{array}{lll}v_{1} & v_{2} & v_{3}\end{array}\right]$ is constrained to lie on the unit sphere (where without loss of generality, we consider their appropriately scaled versions). Hence, $\Sigma_{1}$ is locally reachable in its solution manifold at almost all points contained within.

As $\Sigma_{1}$ is not locally reachable, it is not in minimal form. There is an obvious transformation that will bring it to minimal form. Namely, as $\left[v_{1} v_{2} v_{3}\right]$ lies on the unit sphere, one can take $v_{3}= \pm \sqrt{1-v_{1}^{2}-v_{2}^{2}}$ and substitute it into the right-hand side of (2). The equation for $\dot{\mu}_{z}$ will no longer be needed, and the number of equations will decrease from 5 to 4 .

Going back to the original formulation of $\Sigma_{1}$, although it is nonlinear, the non-linearity is "soft". The $f(v, t)$ term can be written as $f(v, t)=A_{z}(t) v(t)$. Here, $A_{z}(t)$ is a time-varying matrix that depends on $z(t)$, the cantilever position. This is a re-formulation that conveniently ignores the fact that $z$ is in the state vector $v(t)$. However, $z$ is a quantity that is readily available to us, as it is what we observe. There are, however, non-idealities that are present. Firstly, we do not observe $z$ continuously, but only a sampled version. Secondly, the samples are corrupted by noise. If the sampling frequency $f_{s}$ is sufficiently high relative to the bandwidth of $z, z(t)$ will be a slowly-changing signal in each sampling interval $\left[t_{i}, t_{i+1}\right)$, and can be approximated by a constant. Moreover, an estimator $\hat{z}$ can be employed instead of the noisy samples $x[i]$. This provides a "cleaner" version of $z\left(t_{i}\right)$. If the Signal to Noise Ratio (SNR) is sufficiently low, we should have $\hat{z}\left(t_{i}\right) \approx z\left(t_{i}\right)$.

\section{MODEL DETECTION}

\subsection{State estimation of $\Sigma_{1}$ from noisy samples}

Assume that the sampling frequency $f_{s}$ is sufficiently high, so that $z(t)$ in each sampling interval $\left[t_{i}, t_{i+1}\right)$ can be approximated as $z(t) \approx \hat{z}\left(t_{i}^{+}\right)$. We use the notation that appears in [4], where $t_{i}^{-}$denotes the time right before the $i$ th observation is available, and so $\hat{v}\left(t_{i}^{-}\right)$, the estimator of $v\left(t_{i}\right)$, does not incorporate information from $z\left(t_{i}\right)$. Similarly, $t_{i}^{+}$is the time right after the $i$-th observation, and $\hat{v}\left(t_{i}^{+}\right)$has information from $z\left(t_{i}\right)$ incorporated into it. The time dependence of $A_{z}(t)$ lies in $B_{1}(t)$. Assume that $B_{1}(t)$ is sufficiently slowly varying relative to $f_{s}$, so that we can approximate $B_{1}(t) \approx B_{1}\left(t_{i}\right) \forall t \in\left[t_{i}, t_{i+1}\right)$. Then, it is possible to approximate $A_{z}(t)$ for $t \in\left[t_{i}, t_{i+1}\right)$ by $A_{z}(t) \approx$ $A_{\hat{z}\left(t_{i}^{+}\right), B_{1}\left(t_{i}\right)}$. Thus, the nonlinear system $\Sigma_{1}$ can be approximated as a piece-wise Linear Time Invariant (LTI) system, which we shall denote as $\Sigma_{1 i}$. It is then possible to apply the mixed-time Kalman Filter to $\Sigma_{1 i}$ in each sampling interval. Instead of propagating the state estimate through $\Sigma_{1 i}$ however, we shall take a page from the Extended Kalman Filter (EKF) algorithm and propagate the state estimate through the original system $\Sigma_{1}$.

We call this technique piece-wise constant Kalman Filtering (PCKF) for mixed-time systems. Note that only the propagation equations have changed, as compared to the normal mixed-time $\mathrm{KF}$ equations [4]. The measurement update equations remain the same. The piece-wise constant $\mathrm{KF}$ can be viewed as a 0th order mixed-time EKF. The difference between our proposed filter and the normal mixedtime EKF lies in the propagation of the error covariance ma- 
trix $P(t)$ through time [9].

Define $\hat{v}\left(t / t_{i-1}\right)$ for $t \in\left[t_{i-1}, t_{i}\right)$ as the state estimate (conditional mean) of $v(t)$ conditioned on all the observations up until $z\left(t_{i-1}\right)$. Then, $\hat{v}\left(t_{i}^{-}\right)=\hat{v}\left(t_{i} / t_{i-1}\right)$, where $\hat{v}\left(t / t_{i-1}\right)$ is obtained by solving the differential equation $\dot{\hat{v}}\left(t / t_{i-1}\right)=f(\hat{v}, t)$ with $\hat{v}\left(t_{i-1} / t_{i-1}\right)=\hat{v}\left(t_{i-1}^{+}\right)$. The rest of the propagation equations for the $(i-1)$ th sampling interval $\left[t_{i-1}, t_{i}\right)$ are as follows:

$$
\begin{aligned}
\hat{v}\left(t_{i}^{-}\right) & =\hat{v}\left(t_{i} / t_{i-1}\right) \\
P\left(t_{i}^{-}\right) & =\Phi\left(t_{i}, t_{i-1}\right) P\left(t_{i-1}^{+}\right) \Phi^{T}\left(t_{i}, t_{i-1}\right)+ \\
& \int_{t_{i-1}}^{t_{i}} \Phi\left(t_{i}, \tau\right) Q \Phi^{T}\left(t_{i}, \tau\right) d \tau \\
\Phi\left(t_{i}, t_{i-1}\right) & =\exp \left[A_{\hat{z}\left(t_{i-1}^{+}\right), B_{1}\left(t_{i-1}\right)}\left(t_{i}-t_{i-1}\right)\right]
\end{aligned}
$$

\subsection{Post-FM energy detector}

As was mentioned in the introduction, a straight-forward method to detect the presence of the spin moment is to pass $z(t)$ through a FM demodulator. Let $s(t)$ be the output of the FM demodulator. Assume that $s(t)$ under $H_{1}$ will be a periodic telegraph signal with random transitions and additive WGN. This suggests the use of an energy detector. Under high SNR, the energy of $s(t)$ under $H_{1}$ will be greater than that under $H_{0}$. So. the test statistic is $T^{f m}=$ $\sum_{i=1}^{N} s^{2}[i] \underset{H_{0}}{\stackrel{H_{1}}{\gtrless}} \gamma$.

\subsection{Dual KF detector}

An electron spin detector can be constructed by using a dual KF setup. See Figure 1 . Since $\Sigma_{0}$ is linear, the normal mixed-time $\mathrm{KF}$ is optimal in the sense that it minimizes the Mean Squared Error (MSE) of the residuals. Denote by KF0 the KF matched to $\Sigma_{0}$ and $\mathrm{KF} 1$ the piece-wise constant $\mathrm{KF}$ matched to $\Sigma_{1}$. Suppose for the moment that $\Sigma_{0}$ and $\Sigma_{1}$ are both linear systems. Let $\eta_{0}[i]$ and $\eta_{1}[i]$ be the residuals produced by $\mathrm{KF} 0$ and $\mathrm{KF} 1$ respectively. Then, if the observations $x[i]$ are generated by $\Sigma_{k}$, the residual $\eta_{k}[i]$ will be a zero-mean white i.i.d. sequence. The resulting likelihoodratio test statistic can be simplified to:

$$
T=\sum_{i=1}^{N} \frac{\eta_{0}^{2}[i]}{\operatorname{var}\left(\eta_{0}[i]\right)}-\sum_{i=1}^{N} \frac{\eta_{1}^{2}[i]}{\operatorname{var}\left(\eta_{1}[i]\right)} \underset{H_{0}}{\stackrel{H_{1}}{\gtrless}} \gamma
$$

Even though $\Sigma_{1}$ is not linear, we shall nevertheless invoke the piece-wise constant assumption and apply the decision rule above. The KFs need to be initialized with an initial state vector and error covariance matrix. Under high

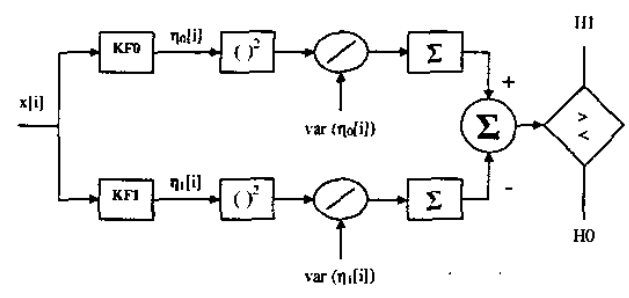

Fig. 1. Electron spin detector: dual Kalman Filter approach

SNR conditions, $z(0)$ and $\hat{z}(0)$ are approximately known. However, the initial spin moment $\vec{\mu}(0)$ is not known, and as it is equally likely to lie anywhere on the unit sphere, $E[\vec{\mu}(0)]=\overrightarrow{0}$. Since $\mathrm{KF} 1$ is sensitive to $\vec{\mu}(0)$, an attempt is made to guess at $\vec{\mu}(0)$. We shall apply the Generalized Likelihood Ratio (GLR) principle, which entails replacing $\vec{\mu}(0)$ with its Maximum Likelihood (ML) estimate. The lower KF1 branch in the electron spin detector is replaced by a filter bank of $p$ KFls, each initialized with a different $\vec{\mu}(0)$. Denote by $\vec{\mu}_{k}(0)$ the value of $\vec{\mu}(0)$ with which the $k$ th KF1 is initialized. The minimum output value of all $p$ KFls will be selected and compared with the output of KF0. By doing this, (4) is modified to be:

$T^{g l r}=\sum_{i=1}^{N} \frac{\eta_{0}^{2}[i]}{\operatorname{var}\left(\eta_{0}[i]\right)}-\min _{1 \leq k \leq p}\left\{\sum_{i=1}^{N} \frac{\eta_{1, k}^{2}[i]}{\operatorname{var}\left(\eta_{1}[i]\right)}\right\} \underset{H_{0}}{\stackrel{H_{1}}{\gtrless}} \gamma$

The values of $\operatorname{var}\left(\eta_{0}[i]\right)$, the variance of $\eta_{0}[i]$ under $H_{0}$, can be obtained from KF0. However, $\operatorname{var}\left(\eta_{1}[i]\right)$ cannot be so easily computed. We can compute an empirical approximation to $\operatorname{var}\left(\eta_{1}[i]\right)$, or we can approximate $\operatorname{var}\left(\eta_{0}[i]\right) \approx$ $\operatorname{var}\left(\eta_{1}[i]\right)$. In the latter case, the LRT test (4) becomes an energy test of the form: $T_{e}=\sum_{i=1}^{N} \eta_{0}^{2}[i]-\sum_{i=1}^{N} \eta_{1}^{2}[i] \gtrless \gamma$. An analogous form exists for the GLR-LRT test (5).

\section{SIMULATION RESULTS}

In simulations that were conducted, 4 different parameter sets with different values of $G$ and $k$ (the spring constant of the cantilever) were considered. $G=2 \mathrm{G} / \AA$ for parameter sets I and II, whereas $G=10 \mathrm{G} / \AA$ for III and IV; $k=10^{-3}$ $\mathrm{N} / \mathrm{m}$ for I and III, and $k=10^{-4} \mathrm{~N} / \mathrm{m}$ for II and IV. Some of the other relevant simulation parameters are as follows: $f_{s}=250 \mathrm{kHz}, \omega_{0}=10 \mathrm{kHz}, \sigma^{2}=10^{-22}, T_{f}=0.6 \mathrm{~K}$ (noise temp. of fundamental mode of cantilever).

The piecewise constant KF performed better than the mixed-time EKF in simulations that were conducted. The result of applying the PCKF and EKF to a simulation from parameter set IV is illustrated in Figure 2 below. The overall Mean Squared Error (MSE) of $\hat{\mu}_{z}$ for the PCKF was $6.823 \times$ 
$10^{-24}$, while for the EKF, it was $1.604 \times 10^{-20}$.

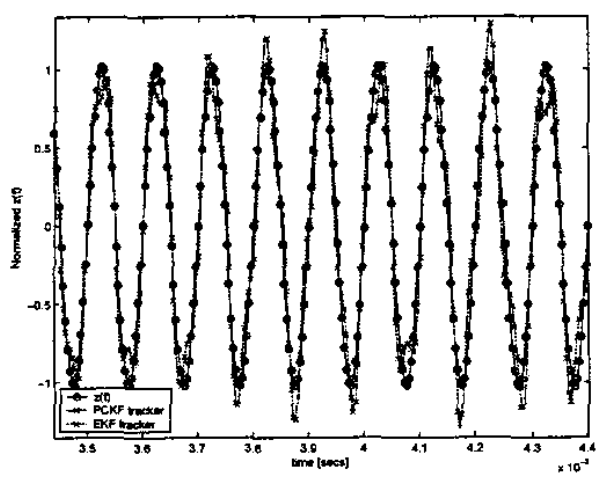

Fig. 2. Comparison of the mixed-time EKF and the PCKF in tracking $z(t)$ under $H_{1}$

We implemented detector test (5) with $p=2$. Each Receiver Operating Characteristic (ROC) curve was generated from 100 to 120 simulation trials ( 100 for I, $\mathrm{II}$ and 120 for III, IV); each trial was $25 \mathrm{~ms}$ in length. The dual $\mathrm{KF}$ spin detector performed better than the post-FM energy detector in all four parameter sets studied. Parameter set $I$ resulted in the worst performance, while IV resulted in the best performance. Their ROC curves are given in Figures $3 a$ and $3 b$ respectively, with the other two cases being omitted due to a lack of space.

\section{CONCLUSION}

We have developed a modified form of the Kalman Filter for the state estimation of a soft nonlinear system. The modified estimator, known as the piece-wise constant Kalman Filter, can be viewed as a 0th order EKF. With this, we constructed an electron spin detector that uses a dual KF setup derived from the residual LRT test. Under the constraints of a small sampling frequency and observation time window, the dual $\mathrm{KF}$ detector out-performs the post-FM energy detector.

\section{REFERENCES}

[1] B. C. Stipe et al., "Magnetic dissipation and fluctuations in individual nanomagnets measured by ultrasensitive cantilever magnetometry," Physical Review Letters, vol. 86, no. 13, pp. 2874-2877, 2001.

[2] K. Wago et al., "Magnetic resonance force detection and spectroscopy of electroon spin in phosphorusdoped silicon," Review of scientific instruments, vol. 68, no. 4, pp. 1823-1826, 1997.

[3] C. Y. Yip et al., "Baseband detection of bistatic electron

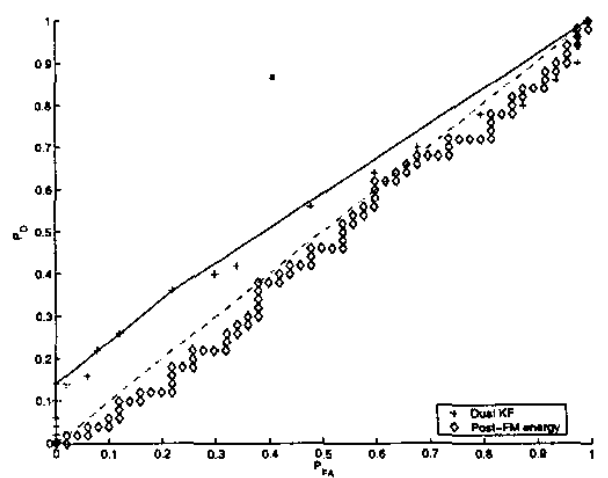

(a) Parameter set I ( $\left.G=2 \mathrm{G} / \AA, k=10^{-3} \mathrm{~N} / \mathrm{m}\right)$

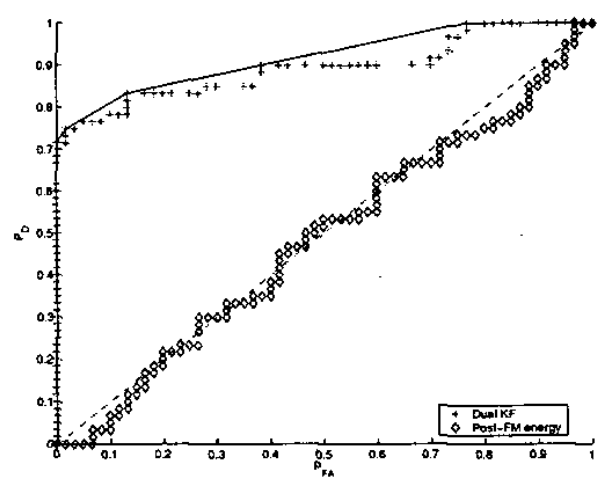

(b) Parameter set IV $\left(G=10 \mathrm{G} / \AA, k=10^{-4} \mathrm{~N} / \mathrm{m}\right)$

Fig. 3. Simulated ROC curve of the dual KF detector using the residual LRT test

spin signals in Magnetic Resonance Force Microscopy (MRFM)," ArXiv: Quantum Physics, vol. 0307, 2003.

[4] P. Maybeck, Stochastic models, estimation and control: Volume 1, Academic Press, Orlando, Florida, 1979.

[5] C. K. Chui and G. Chen, Kalman Filtering with realtime applications, Springer-Verlag, Berlin, 1987.

[6] D. Rugar and R. Budakian, "Classical dynamics of a spin interacting with a MRFM cantilever," Tech. Rep., IBM, 2002.

[7] M. Vidyasagar, Nonlinear Systems Analysis, Prentice Hall, Englewood Cliffs, New Jersey, 1993.

[8] A. Isidori, Nonlinear Control Systems: An Introduction, Springer-Verlag, Berlin, 1985.

[9] K. S. Miller and D. M. Leskiw, An introduction to Kalman Filtering with applications, Robert E. Krieger Pub. Co., Malabar, Florida, 1987. 